Rev. Int. Contam. Ambie. 36 (1) 165-175, 2020

DOI: 10.20937/RICA.2020.36.53209

\title{
LA ACTIVIDAD MINERA DEL SIGLO XX EN EL VALLE DE OAXACA: RIESGOS DE SALUD PÚBLICA DE HOY
}

\author{
Mining activity in the 20th century in the Oaxaca Valley: Today's public health risks \\ Hugo Adalberto VON THADEN UGALDE ${ }^{1 *}$, Celerino ROBLES $^{1}$ y \\ Mario Enrique FUENTE CARRASCO ${ }^{2}$
}

\begin{abstract}
${ }^{1}$ Centro Interdisciplinario de Investigación para el Desarrollo Integral Regional, Unidad Oaxaca, Instituto Politécnico Nacional, Hornos 1003, Col. Noche Buena, 71230 Santa Cruz Xoxocotlán, Oaxaca, México

${ }^{2}$ Universidad de la Sierra Juárez, Instituto de Estudios Ambientales, Avenida Universidad S/N, Ixtlán de Juárez, Oaxaca, C.P. 68725

*Autor para correspondencia: hugo.von.ugalde@gmail.com
\end{abstract}

(Recibido: abril 2018; aceptado: febrero 2019)

Palabras clave: jales, metales pesados, San Jerónimo Taviche

\section{RESUMEN}

El abandono de la minería es un tema que ha pasado inadvertido tanto en el debate nacional desde mediados del siglo XX como en la normatividad. Los jales, apilamientos de rocas molidas que quedan después de que los minerales de interés son extraídos, tienen un impacto socio-ambiental. Sin embargo, no se han definido las responsabilidades de las empresas, del Estado y, en general, de la sociedad civil frente a los efectos contaminantes de los jales. El presente estudio evalúa la situación socio-ambiental en San Jerónimo Taviche, Oaxaca, México, donde empresas trasnacionales generaron grandes volúmenes de jales. Para evaluar la parte ambiental se analizaron 10 muestras de cuerpos de agua y 13 muestras de suelo en las que se cuantificó, mediante espectrofotometría de absorción atómica, el nivel de concentración de los metales pesados totales cadmio, cobre, mercurio, plomo y zinc. Para evaluar el aspecto social se realizaron entrevistas semiestructuradas a los mineros, alumnos de la telesecundaria y médicos del centro de salud, donde se registraron las condiciones laborales, los procesos de extracción de metales pesados y las enfermedades más comunes en los mineros. Se encontró que las concentraciones de algunos metales sobrepasan los límites establecidos por la normatividad mexicana vigente; además, las entrevistas muestran que las personas no conocen los riesgos ante el problema de la contaminación proveniente de los jales. $\mathrm{La}$ presente investigación permite entender la visión del riesgo sanitario y ambiental que representan los jales abandonados para la población.

Key words: mine tailings, heavy metals, San Jerónimo Taviche

\begin{abstract}
The abandonment of mining is a topic that has gone unnoticed in the national debate since the mid-twentieth century, as well as in the normativity. The tailings, stacks of ground rocks that remain after the minerals of interest are extracted from the rocks, have a socio-environmental impact. However, the responsibilities of companies, the
\end{abstract}


State and, in general, of civil society against the contaminating effects of tailings have not been defined. The present study evaluates the socio-environmental situation in San Jerónimo Taviche, Oaxaca, Mexico, where transnational companies generated large volumes of tailings. To evaluate the environmental part, 10 samples of water bodies and 13 soil samples were analyzed in which the concentration level of the total heavy metals (cadmium, copper, mercury, lead and zinc) was quantified by atomic absorption spectrophotometry. To evaluate the social aspects, semi-structured interviews were conducted with the miners, telesecundaria students and doctors from the health center, where the working conditions, the extraction processes of the heavy metals and the most common diseases in the miners were registered. It was found that the concentrations of some metals exceed the limits established by current Mexican normativity; in addition, interviews showed that the population is unaware of the health risks arising from the tailings. The present investigation allows understanding the vision of the sanitary and environmental risks that the abandoned tailings represent for the population.

\section{INTRODUCCIÓN}

En México, las actividades mineras han adquirido una amplia relevancia en el debate nacional. Por una parte, se destaca su contribución al aumento del producto interno bruto dentro de la inversión extranjera directa (Guevara 2016). También se ha apreciado el alto potencial de México como exportador de minerales, pero con un alto costo socioambiental (Delgado 2010). Asimismo, se destacan avances en materia legislativa y regulatoria (INECC 2016).

En contraparte, algunos autores se han enfocado a analizar el tema de la generación de conflictos distributivos, tanto económicos como socioambientales (Composto 2012, Azamar y Ponce 2015). En este sentido, por ejemplo, enfatizan la importancia de nuevos derechos (humanos y de pueblos indígenas) como mecanismos de la sociedad para definir la pertinencia o improcedencia de proyectos mineros en sus territorios. El estado de Oaxaca no ha sido la excepción en este debate. Dada su riqueza mineral y su alta diversidad biológica y cultural, el tema ha sido intensamente discutido. El proyecto minero de Capulálpam de Méndez en la región Sierra Norte, y el de San José del Progreso en la región Valles Centrales, son dos casos ampliamente analizados y discutidos del debate estatal (Sigüenza 1996, Fuente y Barkin 2013, Azamar y Ponce 2014).

El análisis (político y académico) realizado sobre el papel de la minería presenta un contrasentido. Por un lado, existe una amplia discusión sobre las implicaciones (positivas y negativas) del desarrollo de la industria minera en el siglo XXI. Por otro lado, las implicaciones y riesgo para la salud de la actividad minera abandonada en el siglo XX está ausente del debate nacional y estatal. Hay evidencias de la existencia de reservorios de residuos de minería (jales) a lo largo de la República Mexicana, pero no existen mecanismos jurídicos ni estudios que valoren esta situación como potenciales generadores de riesgo. En este sentido, se propone abrir una línea de investigación que permita generar un mayor conocimiento sobre la relación entre un problema de contaminación heredado del siglo pasado $(\mathrm{XX})$ para las generaciones presentes y futuras (XXI). Los estudios de Martín y Gutiérrez (2010) proporcionan antecedentes relevantes en esta exploración al señalar que las técnicas de flotación y cianuración para extraer grandes cantidades de metales preciosos han sido las causantes de la generación de enormes volúmenes de jales.

Como se puede evidenciar, se trata de un tema relevante no sólo desde el punto de vista académico, sino también (y sobre todo) desde la óptica de la política pública. No es un estudio histórico-académico orientado hacia la evaluación retrospectiva del impacto que tuvo la actividad minera del siglo pasado; es un asunto preventivo de salud pública y ambiental. Específicamente, se trata de identificar los riesgos a la salud para las generaciones presentes y futuras de los habitantes de comunidades que albergaron la actividad minera. Y concretamente se pretende caracterizar la exposición de los habitantes a la presencia y dispersión de metales pesados en los llamados jales como reservorios de residuos industriales. Desde el punto de vista de política pública y ambiental se evidencia la necesidad de contar con información empírica de problemas de contaminación sui generis (los jales) como insumos para desarrollar e incluir nuevos principios ambientales en los instrumentos jurídicos. El referente empírico se desarrolla en la población de San Jerónimo Taviche, ubicada en la región de los Valles Centrales del estado de Oaxaca.

A partir del análisis de la situación local, se propone una interrogante desde el ámbito de las 
responsabilidades públicas: ¿quién es responsable de atender este problema de contaminación? La falta de atención a este asunto de salud pública puede considerarse jurídicamente como una violación a los derechos humanos conforme al artículo cuarto de la Constitución mexicana, en el que explícitamente se indica la responsabilidad del Estado para mantener un ambiente saludable.

El abordaje metodológico tiene un carácter interdisciplinario. Abarca tanto aspectos de tipo técnico para identificar los contenidos de metales pesados en los jales (y en el suelo y agua en contacto con ellos), como de tipo social, entre los cuales se exploran algunos componentes sociodemográficos. Estos temas se exponen desde tres ejes de análisis: en el primero se proporcionan algunos antecedentes del sector minero en el periodo comprendido entre el inicio de la época colonial y mediados del siglo XX; en el segundo eje se hace una descripción técnica del potencial de contaminante de los jales, y su relación con el tema de los riesgos a la salud; en el tercero, se realiza una descripción del referente empírico (San Jerónimo Taviche) y su actividad minera durante el siglo XX. Finalmente, se formula una hipótesis de trabajo sobre el riesgo potencial a la salud de los habitantes de la población (pasado, presente y futuro) de los jales ubicados en su territorio.

\section{CONTEXTO}

\section{Antecedentes históricos de la actividad minera en Oaxaca y en Taviche}

El territorio oaxaqueño y la región de los Valles Centrales en particular, poseen una amplia riqueza de recursos minerosl. Principalmente se extraen oro y plata, de acuerdo con el Sistema Integral de Administración Minera (SIAM 2015). Por ello, la historia y situación actual de la entidad no pueden comprenderse de forma amplia sin la inclusión del análisis del papel de la minería. Desde la época prehispánica, los zapotecas cribaban el fondo arenoso de sus arroyos para extraer minerales preciosos, mientras que en la colonia son frecuentes las reseñas en las que se destaca la importancia de los Valles de Oaxaca en las encomiendas de oro y los descubrimientos de grandes yacimientos de plata (Sigüenza 1996). En esta historia del desarrollo minero, la población de San Jerónimo Taviche es emblemática, con una alta importancia durante el siglo XX en la extracción de oro y plata (Sánchez 1993).

En los inicios de la explotación minera colonial en San Jerónimo Taviche, la extracción de minerales, así como la posesión de los medios de producción, estuvieron bajo control de la monarquía española. Se estableció una población creciente de zapotecas, siendo los hombres y menores de edad quienes trabajaban en las minas. A raíz de la actividad minera, y sin una fundación formal, se establece la población de San Jerónimo Taviche, probablemente en el año 1600. Para finales del siglo XIX se consolidó un nuevo sistema económico en la población, intrduciéndose la primera empresa de capital estadounidense: American Smelting and Refining Company (ASARCO). Los mineros que en esa época laboraban en las minas eran principalmente zapotecas. Casi al finalizar el siglo XIX, el presidente Porfirio Díaz introdujo el Ferrocarril Mexicano del Sur, que comunicó a la capital de la República con la ciudad de Oaxaca, derivando desde esta última un ramal dirigido expresamente a San Jerónimo Taviche, con la finalidad de hacer más ágil y costeable el transporte de los minerales extraídos (Sigüenza 1996).

Los registros de las condiciones laborales son escasos, en su mayoría relatos. Algunos mineros que trabajaron en ASARCO fueron los padres y abuelos de los mineros que, durante la primera mitad del siglo XX, trabajaron en las empresas Minerales de Taviche y Minerales de Oaxaca, ambas de capital canadiense. En todas estas empresas, las condiciones laborales eran precarias.

\section{Los jales como reservorios de metales pesados}

En México, la industria minera ha removido grandes cantidades de rocas que han sido pulverizadas para la extracción de oro y plata por medio de procesos como la cianuración, que fue uno de los métodos de extracción más utilizados (Salinas et al. 2004). En dicho proceso se combinan la oxidación a presión y el anión cianuro (Parga y Carrillo 1996). La gran maquinaria minera generó una enorme cantidad de jales, residuos de la separación mineral, que a menudo contienen metales pesados (arsénico, cadmio, mercurio, manganeso, cobre, plomo, y zinc) que se dispersan con una gran facilidad en el ambiente (Teodoro 2014).

\section{Metales pesados e impacto para la salud}

Los metales pesados son un riesgo potencial para la salud. Ocasionan un estrés oxidativo por la generación de peróxido de hidrógeno y radicales libres que desencadenan mutaciones del ADN y alteran las estructuras de carbohidratos, proteínas y lípidos (Xu et al. 2008). Algunos de los metales pesados más tóxicos son plomo y mercurio. 
Recientemente, Bjørklund et al. (2017) reportaron que las especies mono-metiladas del arsénico son potentes inductores de lesiones bioquímicas que, por su toxicidad y potencial, pueden causar cáncer de piel, pulmón, hígado y riñón. En el mismo sentido, Ordóñez et al. (2003) evaluaron a 750 niños de Ciudad Juárez (Chihuahua, México) que residían cerca de una fundición en El Paso, Texas. Los niños que habitaban a menos de una milla de la fundición presentaron una media de $38.66 \mu \mathrm{g} / 100 \mathrm{~mL}$ de plomo en sangre, a diferencia de los que habitaban una distancia de 6.1 a 8 millas, con una media de $27.71 \mu \mathrm{g} / 100 \mathrm{~mL}$ de plomo en sangre. Carrizales et al. (2006) registraron que el 90 $\%$ de las muestras de suelo de la población de Morales en el estado de San Luis Potosí (México) tuvieron valores mayores a $400 \mathrm{mg} / \mathrm{kg}$ de plomo y a $100 \mathrm{mg} / \mathrm{kg}$ de arsénico. Además, mostraron sus impactos en la población infantil de la siguiente forma: el grupo de niños de 3 a 6 años de edad fue el más vulnerable, ya que el $90 \%$ presentaban una concentración mayor de $10 \mu \mathrm{g} /$ $\mathrm{dL}$ de plomo en sangre, mientras que los niños de 8 y 9 años presentaron la mayor exposición al arsénico, con una media de $80 \mu \mathrm{g} / \mathrm{g}$ de creatinina urinaria. Peters et al. (2014) han mostrado que la concentración de creatinina urinaria está positivamente asociada con el porcentaje de ácido dimetilarsinico $\left(\mathrm{C}_{2} \mathrm{H}_{7} \mathrm{AsO}_{2}\right)$ y negativamente con arsénico inorgánico, ambos excretados en orina.

Diversos autores han destacado los riesgos de salud por presencia de metales pesados. Canfield et al. (2003) destacan un dato epidemiológico relevante: los niños son los más sensibles al plomo, y los deterioros neurológicos comienzan a partir de $10 \mu \mathrm{g} / \mathrm{dL}$ de plomo en sangre. En el mismo sentido, Needleman (2004) identifica que niveles de plomo superiores 60 $\mu \mathrm{g} / \mathrm{dL}$ desencadenan en niños síntomas como dolores abdominales, artralgia y encefalopatías. El plomo se fija en la superficie de la membrana celular, cuya permeabilidad y transporte activo son alterados. Batool et al. (2017) son contundentes al afirmar que el plomo tiene la capacidad de interactuar con las proteínas al imitar las funciones del calcio.

Los niveles normales de mercurio en sangre rara vez superan los $1.5 \mathrm{mg} / \mathrm{dL}$ (Graeme y Pollack 1998). Sin embargo, estudios epidemiológicos en las Islas Feroe han mostrado que se genera un desarrollo neurológico deficiente luego de la exposición a largo plazo a pequeñas cantidades de metilmercurio (Grandjean et al. 1997). Genchi et al. (2017) mencionan que dichas concentraciones afectan la función mitocondrial, disminuyen la síntesis de ATP, reducen el glutatión y aumentan la peroxidación de fosfolípidos, proteínas y ADN. Sus efectos se relacionan con la generación de enfermedades cardiacas y cerebrales.

\section{Definición de riesgo e importancia del principio precautorio}

La evaluación del riesgo es un instrumento que identifica las posibles consecuencias negativas de algún evento (Alfie 2007). A menudo, las investigaciones no llegan a detectar algún tipo de riesgo. El principio precautorio indica que no necesariamente deben existir pruebas concluyentes sobre daños potenciales para establecer la normatividad necesaria para prevenir riesgos perjudiciales (Cózar 2001).

\section{MATERIALES Y MÉTODOS}

El lugar de estudio es el municipio de San Jerónimo Taviche, pertenece al distrito de Ocotlán, en el estado de Oaxaca, México. Se encuentra aproximadamente $46 \mathrm{~km}$ al sur de la ciudad de Oaxaca, en las coordenadas $16^{\circ} 42$ ' 48.7' $\mathrm{N}, 96^{\circ} 35^{\prime} 39.5^{\prime}$ ' O, con una altitud media de $1700 \mathrm{msnm}$.

\section{Técnica}

\section{Estudios de contenido de metales pesados}

Las muestras compuestas de suelo y jales se extrajeron a una profundidad de $0-15 \mathrm{~cm}$. Para obtener las muestras de agua se hizo una excavación hasta una profundidad de $30 \mathrm{~cm}$ en el caudal del arroyo para acumular el agua (Fig. 1). A las muestras se les agregó ácido nítrico y se refrigeraron a $4{ }^{\circ} \mathrm{C}$ hasta su análisis.

Las muestras de suelo y jal se secaron a $95{ }^{\circ} \mathrm{C}$ hasta peso constante. Para determinar el contenido total de metales pesados (CTMP) se utilizaron $0.5 \mathrm{~g}$ de suelo (tamizado a $2 \mathrm{~mm}$ ) y se realizó la digestión en mezcla de ácido nítrico, peróxido de hidrogeno y agua $\left(\mathrm{HNO}_{3}: \mathrm{H}_{2} \mathrm{O}_{2}: \mathrm{H}_{2} \mathrm{O} ; 3: 2: 10\right)$ durante 30 min en autoclave a $1.02 \mathrm{~atm}$. El filtrado se enrasó a $50 \mathrm{~mL}$ (Moreno 2010). Para la fracción extraíble con ácido dietilentriaminopentaacético (DTPA), desarrollado por Lindsay y Norvell (1978), se utilizaron $10 \mathrm{~g}$ (jal y suelo) y $10 \mathrm{~mL}$ (agua) de muestra por $20 \mathrm{~mL}$ de DTPA (0.005 M DTPA, 0.1 M trietanolamina y $0.01 \mathrm{M}$ cloruro de calcio, con $\mathrm{pH}$ de 7.3). Para las muestras de agua se siguieron las indicaciones de la NOM-001-SEMARNAT-1996 (SEMARNAT 1996).

Tanto las muestras como los blancos se analizaron por triplicado. Las lecturas de metales pesados se realizaron por espectrofotometría de absorción atómica (Perkin Elmer AAnalyst). Se cuantificaron cadmio, cobre, mercurio, plomo y zinc. 


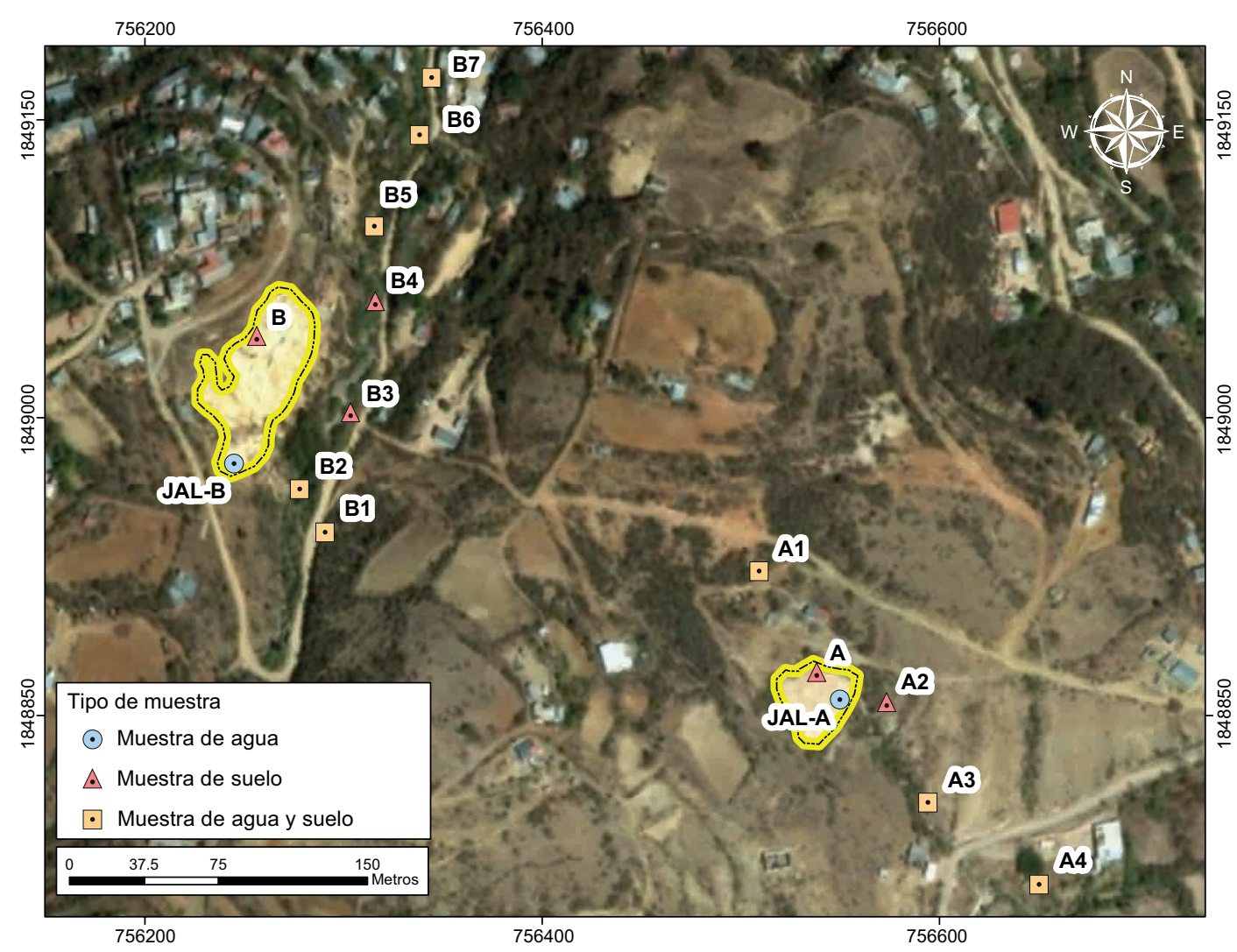

Fig. 1. Localización de los sitios de muestreo en el municipio de San Jerónimo Taviche, Oaxaca, México

\section{Aspectos sociales}

Se realizaron entrevistas semiestructuradas a dos grupos sociales en la población: todos los mineros sobrevivientes que trabajaron en la empresa Minerales de Taviche (siete personas) y 51 estudiantes de la telesecundaria de San Jerónimo Taviche. En el caso de estos últimos, el cuestionario trató de capturar su percepción sobre la problemática ambiental de su comunidad en general y en particular sobre los jales. Fue relevante indagar dos asuntos sobre estos últimos: su contacto en la infancia y los problemas de salud que más los aquejan.

Además, se visitó el centro de salud para conocer cuáles son las las enfermedades que afectan a los habitantes. La finalidad no fue generalizar los problemas de salud de la población, sino tener un panorama similar a un estudio de línea de base.

\section{RESULTADOS Y DISCUSIÓN}

Los resultados de la investigación proporcionan una aproximación a la problemática de salud ambiental asociada a los jales que se presenta en San Jerónimo Taviche.
Los mineros recuerdan de manera clara una mayor incidencia de problemas respiratorios en las personas dedicadas a la extracción. Sólo un pequeño grupo de mineros de 60 a 72 años de edad (promedio = 68 años; desviación estándar $=5.04$ ) sobrevive actualmente en la población. Relatan que estuvieron expuestos directa e indirectamente a mercurio, plomo, cianuro y arsénico, y describen la situación laboral en las empresas que recuerdan personalmente o por relatos de sus abuelos y padres (Cuadro I). La mayor problemática de salud relatada es la relacionada con las vías respiratorias. Perforaban las rocas protegidos únicamente con un paliacate para cubrir las vías respiratorias, por consiguiente, inhalaban cristales de sílice por largos periodos de tiempo. La inhalación constante de estos cristales ocasiona silicosis, cuyo diagnóstico sólo requiere el conocimiento de la historia laboral de exposición a la sílice cristalina (Rees y Murray 2007). Es una enfermedad pulmonar que puede desencadenar cáncer pulmonar, problemas renales y tuberculosis (Martínez et al. 2010).

A partir de la década de 1950, la empresa canadiense Minerales de Taviche generó dos jales a cielo abierto en la población, antes de la publicación de 
CUADRO I. CONDICIONES DE LA MINERÍA EN SAN JERÓNIMO TAVICHE DESCRITA POR SUS MINEROS

\begin{tabular}{|c|c|c|c|c|}
\hline Empresa & $\begin{array}{c}\text { Periodo } \\
\text { aproximado }\end{array}$ & $\begin{array}{l}\text { Minerales que } \\
\text { explotaban }\end{array}$ & $\begin{array}{l}\text { Condiciones } \\
\text { laborales }\end{array}$ & Enfermedades \\
\hline ASARCO & $1900-1910$ & $\begin{array}{l}\text { Se desconoce qué se } \\
\text { extraía porque toda la } \\
\text { roca era transportada en } \\
\text { ferrocarril a las instala- } \\
\text { ciones de la empresa en } \\
\text { Monterrey }\end{array}$ & $\begin{array}{l}\text { No tenían ninguna me- } \\
\text { dida de seguridad, utili- } \\
\text { zaban guaraches de tres } \\
\text { correas y, para proteger- } \\
\text { se las vías respiratorias, } \\
\text { utilizaban un paliacate }\end{array}$ & Problemas respiratorios \\
\hline Minerales de Taviche & 1950 a 1962 & Oro, plata y plomo & $\begin{array}{l}\text { Contaban con guara- } \\
\text { ches de tres correas, un } \\
\text { paliacate y una lámpara } \\
\text { de carburo }\end{array}$ & $\begin{array}{l}\text { La piel se les tornaba } \\
\text { de color amarillo, tosían } \\
\text { mucho, vomitaban sangre } \\
\text { y a las pocas semanas } \\
\text { fallecían }\end{array}$ \\
\hline Minerales de Oaxaca & 1965 a 1993 & Oro y plata & $\begin{array}{l}\text { Les proporcionaban una } \\
\text { lámpara de baterías, } \\
\text { casco de plástico, botas } \\
\text { y guantes }\end{array}$ & Problemas respiratorios \\
\hline
\end{tabular}

ASARCO: American Smelting and Refining Company

la Ley Minera de 1992 y de la NOM-141-SEMARNAT-2003 (SEMARNAT 2003), donde se establece por primera vez el marco normativo de la concesión del aprovechamiento minero y de la planeación y ejecución de las presas de jales, respectivamente.

Son muchas las localidades que tienen jales depositados de manera incontrolada en México. Romero et al. (2008) reportan las concentraciones de metales pesados en las regiones de Santa Bárbara, Chihuahua; Zimapán, Hidalgo, y Taxco de Alarcón, Guerrero. Llegaron a encontrar hasta $6669 \mathrm{mg} / \mathrm{kg}$ de plomo en Santa Bárbara. San Jerónimo Taviche presenta la misma problemática. Los dos jales mencionados tienen un $\mathrm{pH}$ menor a 6 (Teodoro 2014). El pH es el responsable de las reacciones de hidrolisis de los cationes que ocasionan alta movilidad de los metales (RamosGómez et al. 2012). Con un pH bajo se incrementa la adsorción de los iones metálicos a la superficie de los minerales, y a un $\mathrm{pH}$ alto ésta disminuye. En ambos jales, Teodoro (2014) encontró porcentajes bajos de materia orgánica $(<1.3)$, componente que regula la adsorción de iones metálicos (Warren y Haack 2001). La descomposición de materia orgánica es el principal impulsor del carbono orgánico disuelto y la movilidad del metal en el suelo. La movilización del cobre depende del carbono orgánico disuelto, mientras que la movilización del zinc depende principalmente del calcio y en menor medida del carbono orgánico disuelto (Zhao et al. 2007).

En los cuadros II y III se presentan las concentraciones de plomo y mercurio que sobrepasan la referencia total de la NOM-147-SEMARNAT/ SSA1-2004 (SEMARNAT 2004) en los jales y los diferentes sitios de muestreo de suelo (Fig. 1). El sitio A2 presentó la mayor concentración de mercurio y plomo. El jal B y los sitios de muestreo aledaños no muestran contaminación por mercurio. En la muestra A2, la concentración total de plomo fue $29.23 \%$ mayor a la que se reporta en estudios realizados en los jales de Zimapán, Hidalgo (Romero et al. 2008).

No existe una correlación de las concentraciones de metales pesados entre los sitios de muestreo y los jales. Puga et al. (2006) encontraron que no siempre hay homogeneidad en la relación de profundidad, distancia y concentración de los metales pesados, ya que, al carecer de materia orgánica, los jales no favorecen la retención de metales pesados, los cuales se infiltran a los cuerpos de agua con mayor facilidad. Simón et al. (1999) afirman que la infiltración de metales pesados generalmente tiende a disminuir entre 10 y $30 \mathrm{~cm}$ de profundidad en el suelo.

Para corroborar si los metales pesados son transportados a las zonas de acuíferos de la población, se realizaron análisis de agua en los arroyos que se encuentran cercanos a los jales (Fig. 1). El jal B contaminó el agua y suelo del arroyo aledaño con cadmio, cobre y zinc en mayor medida que el jal A. Sin embargo, el jal A contamino más el agua y suelo del arroyo con plomo (Cuadro IV). En los estados de Puebla y Veracruz, Mancilla-Villa et al. (2012) reportan algunos valores mayores a los límites máximos permisibles para plomo, cadmio y 
CUADRO II. CONCENTRACIONES DE METALES PESADOS EN JALES Y SUELO DE ZONAS CIRCUNDANTES CON CTMP

\begin{tabular}{cccccr}
\hline Muestra & $\begin{array}{c}\text { Cadmio } \\
(\mathrm{mg} / \mathrm{kg})\end{array}$ & $\begin{array}{c}\text { Cobre } \\
(\mathrm{mg} / \mathrm{kg})\end{array}$ & $\begin{array}{c}\text { Mercurio } \\
(\mathrm{mg} / \mathrm{kg})\end{array}$ & $\begin{array}{c}\text { Plomo } \\
(\mathrm{mg} / \mathrm{kg})\end{array}$ & $\begin{array}{c}\text { Zinc } \\
(\mathrm{mg} / \mathrm{kg})\end{array}$ \\
\hline A & 0 & 15.929 & 0 & $735.278^{*}$ & 16.922 \\
$\mathrm{~A} 1$ & 0 & 0.622 & 0 & 2.544 & 6.338 \\
$\mathrm{~A} 2$ & 0 & 10.163 & $59.086^{*}$ & $2628.506^{*}$ & 46.982 \\
$\mathrm{~A} 3$ & 0 & 7.164 & 0.035 & $864.564^{*}$ & 105.574 \\
$\mathrm{~A} 4$ & 0 & 2.262 & 0 & 155.238 & 25.206 \\
B & 0 & 5.881 & 0 & 161.385 & 33.759 \\
$\mathrm{~B} 1$ & 0 & 1.187 & 0 & 0 & 8.419 \\
$\mathrm{~B} 2$ & 0 & 1.148 & 0 & 2.196 & 8.353 \\
$\mathrm{~B} 3$ & 0.772 & 4.304 & 0 & 20.825 & 6.338 \\
B4 & 0.284 & 2.838 & 0 & 0 & 203.222 \\
$\mathrm{~B} 5$ & 0.117 & 2.25 & 0 & 0 & 238.143 \\
B6 & 0 & 2.098 & 0 & 0 & 175.747 \\
B7 & 0.073 & 2.206 & 0 & 0 & 125.197 \\
\hline
\end{tabular}

*Valores que sobrepasan la concentración de referencia total de la NOM147-SEMARNAT/SSA1-2004 (SEMARNAT 2004)

CTMP: contenido total de metales pesados

CUADRO III. CONCENTRACIONES DE METALES PESADOS EN JALES Y SUELO DE ZONAS CIRCUNDANTES CON DTPA

\begin{tabular}{lccccr}
\hline Muestra & $\begin{array}{c}\text { Cadmio } \\
(\mathrm{mg} / \mathrm{kg})\end{array}$ & $\begin{array}{c}\text { Cobre } \\
(\mathrm{mg} / \mathrm{kg})\end{array}$ & $\begin{array}{c}\text { Mercurio } \\
(\mathrm{mg} / \mathrm{kg})\end{array}$ & $\begin{array}{c}\text { Plomo } \\
(\mathrm{mg} / \mathrm{kg})\end{array}$ & $\begin{array}{c}\text { Zinc } \\
(\mathrm{mg} / \mathrm{kg})\end{array}$ \\
\hline $\mathrm{A}$ & $\mathrm{N} / \mathrm{D}$ & 0.112 & 0 & $\mathrm{~N} / \mathrm{D}$ & 6.495 \\
$\mathrm{~A} 1$ & $\mathrm{~N} / \mathrm{D}$ & 0.236 & 0 & 0.730 & 0.682 \\
$\mathrm{~A} 2$ & $\mathrm{~N} / \mathrm{D}$ & 5.340 & 0 & $776.506^{*}$ & 49.016 \\
$\mathrm{~A} 3$ & $\mathrm{~N} / \mathrm{D}$ & 4.433 & 0 & $402.332^{*}$ & 13.182 \\
$\mathrm{~A} 4$ & $\mathrm{~N} / \mathrm{D}$ & 1.227 & 0 & $452.941^{*}$ & 3.114 \\
$\mathrm{~B}$ & $\mathrm{~N} / \mathrm{D}$ & $\mathrm{N} / \mathrm{D}$ & 0 & 79.939 & 6.284 \\
$\mathrm{~B} 1$ & $\mathrm{~N} / \mathrm{D}$ & $\mathrm{N} / \mathrm{D}$ & 0 & 6.522 & 3.169 \\
$\mathrm{~B} 2$ & $\mathrm{~N} / \mathrm{D}$ & $\mathrm{N} / \mathrm{D}$ & 0 & 7.483 & 3.169 \\
$\mathrm{~B} 3$ & 2.562 & 1.244 & 0 & 8.005 & 357.330 \\
$\mathrm{~B} 4$ & 1.658 & 1.728 & 0 & 4.800 & 222.715 \\
$\mathrm{~B} 5$ & 1.359 & 0.881 & 0 & 6.667 & 111.716 \\
$\mathrm{~B} 6$ & 0.685 & 1.504 & 0 & 6.478 & 95.264 \\
$\mathrm{~B} 7$ & 0.665 & 1.541 & 0 & 6.619 & 95.264 \\
\hline
\end{tabular}

*Valores que sobrepasan la concentración de referencia total de la NOM-147-SEMARNAT/SSA1-2004 (SEMARNAT 2004) N/D: valores no determinados, ya que no alcanzan el límite inferior de detección

DTPA: ácido dietilentriaminopentaacético

mercurio en agua utilizada para riego. Las concentraciones promedio de los metales respectivos fueron de $0.008,0.01$ y $0.005 \mathrm{mg} / \mathrm{L}$. Las concentraciones de estos metales en los cuerpos de agua de Taviche son mayores (Cuadro IV). Esta diferencia se debe a la contaminación recibida desde los jales depositados de forma incontrolada en la comunidad.
CUADRO IV. CONCENTRACIÓN DE METALES PESADOS EN CUERPOS DE AGUA

\begin{tabular}{llccc}
\hline Muestra & $\begin{array}{c}\text { Cadmio } \\
(\mathrm{mg} / \mathrm{L})\end{array}$ & $\begin{array}{c}\text { Cobre } \\
(\mathrm{mg} / \mathrm{L})\end{array}$ & $\begin{array}{c}\text { Plomo } \\
(\mathrm{mg} / \mathrm{L})\end{array}$ & $\begin{array}{c}\text { Zinc } \\
(\mathrm{mg} / \mathrm{L})\end{array}$ \\
\hline Primaria & 0 & 0.027 & $0.725^{*}$ & 0.576 \\
Tanque & 0 & 0.004 & $0.725^{*}$ & 0.504 \\
A1 & 0 & 0.004 & $0.725^{*}$ & 0.084 \\
A3 & 0.033 & 0.117 & $2.207^{*}$ & 6.127 \\
A4 & 0 & 0.004 & $0.725^{*}$ & 0.066 \\
B1 & 0 & 0.207 & $0.59^{*}$ & 1.604 \\
B2 & $4.509^{*}$ & $10.323^{*}$ & 0.456 & $1112.286^{*}$ \\
B5 & 0.16 & 0.681 & 0.281 & $60.426^{*}$ \\
B6 & $0.541^{*}$ & 0.979 & 0.281 & $27.063^{*}$ \\
B7 & 0 & 0.245 & 0.281 & $12.603^{*}$ \\
\hline
\end{tabular}

*Valores que sobrepasan la concentración de referencia total de la NOM-147-SEMARNAT/SSA1-2004 (SEMARNAT 2004)

La contaminación de las corrientes de agua por los jales ha generado un impacto negativo en el ambiente y en la salud de la población, como ya ha sucedido en otros estados de México (Moreno et al. 2012, Ortiz-Ortiz et al. 2017). Esto se refleja en el porcentaje de hijos fallecidos de las mujeres de 15 a 49 años. A nivel nacional el porcentaje es de 2.7; sin embargo, San Jerónimo Taviche presenta un porcentaje del 4.3 (INEGI 2015). El centro de salud de la comunidad informa que las enfermedades que a menudo aquejan a la comunidad (Cuadro V) son problemas estomacales y dolores de cabeza que se 
CUADRO V. PRINCIPALES ENFERMEDADES REPORTADAS EN EL CENTRO DE SALUD DE SAN JERONIMO TAVICHE

\begin{tabular}{ll}
\hline Enfermedad & Posibles causas \\
\hline Diarrea & $\begin{array}{l}\text { Con exposiciones prolongadas al plomo, apare- } \\
\text { cen síntomas gastrointestinales como diarrea }\end{array}$ \\
\hline $\begin{array}{l}\text { Problemas } \\
\text { auditivos }\end{array}$ & $\begin{array}{l}\text { La exposición al plomo puede causar en los } \\
\text { niños problemas de aprendizaje y sordera }\end{array}$ \\
\hline Cefaleas & $\begin{array}{l}\text { En concentraciones de } 40 \text { a } 120 \mu \mathrm{g} / \mathrm{dL}, \text { el plomo } \\
\text { puede causar cefaleas }\end{array}$ \\
\hline
\end{tabular}

Microcefalia Debido a su liposolubilidad, el metilmercurio atraviesa con facilidad la barrera placentaria, y hay riesgo de que el feto presente microcefalia ${ }^{3}$

${ }^{1}$ Ramírez, 2005, ${ }^{2}$ Poma 2008, ${ }^{3}$ Ibrahim et al. 2006

presentan en la época de lluvias. La población consume agua directamente de las tomas domiciliarias y comunales, la cual no recibe tratamientos previos de ningún tipo. Lo anterior es preocupante debido a que el tanque de agua está contaminado con plomo (Cuadro IV). La intoxicación crónica con plomo no presenta síntomas específicos, lo que complica un diagnóstico preciso (Beigel et al. 1998).

Los resultados de la percepción de los jóvenes muestran que sólo el $4 \%$ percibe a los jales como el principal problema ambiental en su comunidad. Asimismo, el $11.32 \%$ de los estudiantes perciben otro tipo de problemas, como la pérdida de la diversidad biológica y la contaminación del agua. Perciben que los cuerpos de agua que presentan problemas de contaminación son los ríos que colindan con los jales. Sorprende que el $34 \%$ identifique a los jales como un atractivo turístico. El $67 \%$ admite que ha jugado en los jales. Esto indica el desconocimiento de la problemática por parte de la población y la escasa información existente. Los alumnos presentan una alta tasa de ausencias a clases a causa de enfermedades. El 27 \% menciona que a menudo presenta problemas estomacales, y el $79 \%$ de este grupo bebe el agua directamente de las tomas públicas o domiciliarias. Las enfermedades respiratorias son las más recurrentes, con un $75 \%$ de incidencia.

La intención de las encuestas no fue encontrar una relación directa entre la concentración de metales pesados en los jales y los problemas de salud. Un estudio de tal naturaleza requiere la aprobación de las autoridades correspondientes y de la población para realizar una serie de actividades metodológicas, entre ellas análisis de sangre. El propósito es iniciar un banco de datos que sirva como un estudio de línea de base. Un punto relevante que arroja la encuesta es el alto nivel de desinformación de la comunidad. Sin embargo, la carencia de datos epidemiológicos no debería impedir la aplicación de ciertas medidas precautorias que eviten riesgos de salud a la población. Las investigaciones de riesgo deben incluirse en la política pública y la toma de decisiones.

En la legislación ambiental mexicana se contemplan algunos instrumentos que promueven la prevención de impactos al ambiente. Para la autorización de las concesiones mineras, el artículo 43 de la ley minera señala que la concesión será suspendida cuando éstas "Pongan en peligro la vida o integridad física de los trabajadores o de los miembros de la comunidad", y "Causen o puedan causar daño a bienes de interés público, afectos a un servicio público o de propiedad privada". La sociedad tiene ahora los instrumentos políticos y normativos necesarios para declinar cualquier concesión minera, pero no existen antecedentes sobre normas ambientales para las actividades mineras abandonadas del periodo histórico citado. Otro asunto más grave: no existen disposiciones normativas para el monitoreo de actividades mineras pasadas, y en su caso, para la aplicación de acciones de mitigación y remediación. Esto genera una pregunta ética: ¿de quién es la responsabilidad de dar seguimiento a este riesgo ambiental y de salud?

Por último, habrá que señalar que de acuerdo con los datos de la Sistema de Administración Minera (SIAM 2015), la empresa minera canadiense Arco Resources Corp tiene la concesión minera en el territorio de San Jerónimo Taviche, y está actualmente en fase de exploración.

\section{CONCLUSIONES}

El plomo es el metal pesado con mayor presencia en las muestras de suelo y agua. Sus valores exceden la normatividad mexicana. La ingesta de este metal pesado por los pobladores representa el principal riesgo.

El desarrollo del presente trabajo posibilitó identificar uno de los principales riesgos en la comunidad. Sin embargo, la actual legislación minera mexicana ha justificado la asignación de concesiones desde una perspectiva de desarrollo sostenible que consiste en la preservación del equilibrio ecológico, protección del ambiente y aprovechamiento de recursos naturales (DOF 2015). El estudio identifica un área débil en materia de política ambiental y del papel del Estado por promover un ambiente sano: los jales de las minas abandonadas. Al otorgar una concesión, se confía en 
que el desarrollo tecnológico reducirá los daños hacia el ambiente. Sin embargo, no existe una legislación que promueva la remediación de la contaminación provocada por empresas mineras que extrajeron los minerales y ya no poseen la concesión.

Esta investigación presenta algunas limitaciones, ya que no profundiza en análisis clínicos en los habitantes. No obstante, es un acercamiento para entender la forma en que los jales contaminan y la percepción de la comunidad. Es necesario continuar con monitoreos y diagnósticos para generar conciencia, y colocar dicha problemática en la agenda pública con carácter de urgencia.

\section{REFERENCIAS}

Alfie M. (2007). Una nueva gestión ambiental: el riesgo y el principio precautorio. Espacio Abierto 16 (2), 209-222.

Azamar A. y Ponce J.I. (2014). Extractivismo y desarrollo: los recursos minerales en México. Probl. Desarrollo 45 (179), 137-158.

DOI: $10.1016 / \mathrm{S} 0301-7036(14) 70144-0$

Azamar A. y Ponce J.I. (2015). El neoextractivismo como modelo de crecimiento en América Latina. Econ. Desarrollo 154 (1), 185-198.

Batool Z., Yousafzai1 N.A., Murad M.S., Shahid S. y Iqbal A. (2017). Lead toxicity and evaluation of oxidative stress in humans. PSM Biol. Res. 2 (2), 79-82.

Beigel Y., Ostfeld I. y Schoenfeld N. (1998). A leading question. N. Engl. J. Med. 339 (12), 827-830.

DOI: 10.1056/NEJM199809173391208

Bjørklund G., Aaseth J., Chirumbolo S., Urbina M.A. y Uddin R. (2017). Effects of arsenic toxicity beyond epigenetic modifications. Environ. Geochem. Hlth. 40 (3), 955-965. DOI: 10.1007/s10653-017-9967-9

Canfield R.L., Henderson C.R., Cory-Slechta D.A., Cox C., Jusko T.A. y Lanphear B.P. (2003). Intellectual impairment in children with blood lead concentrations below $10 \mu$ g per deciliter. N. Engl. J. Med. 348 (16), 1517-1526. DOI: 10.1056/NEJMoa022848

Carrizales L., Razo I., Téllez-Hernández J.I., Torres-Nerio R., Torres A., Batres L.E., Cubillas A.C. y Díaz-Barriga F. (2006). Exposure to arsenic and lead of children living near a copper-smelter in San Luis Potosi, Mexico: Importance of soil contamination for exposure of children. Environ. Res. 101 (1), 1-10.

DOI: 10.1016/j.envres.2005.07.010

Composto C. (2012). Acumulación por despojo y neoextractivismo en América Latina. Una reflexión crítica acerca del Estado y los movimientos socio-ambientales en el nuevo siglo. Astrolabio (8), 323-352.
Cózar J.M. (2005). Principio de precaución y medio ambiente. Rev. Esp. Salud Públic. 79 (2), 133-144.

DOI: $10.1590 / \mathrm{S} 1135-57272005000200003$

Delgado G.C. (2010). América Latina y el Caribe como reservas estratégicas de minerales. En: Ecología política de la minería en América Latina (DelgadoRamos G.C., Ed.). Universidad Nacional Autónoma de México, Ciudad de México, México, pp. 17-57.

DOF (2015). Ley general del equilibrio ecológico y la protección al ambiente. Diario Oficial de la Federación [en línea]. http://biblioteca.semarnat.gob.mx/ janium/Documentos/Ciga/agenda/DOFsr/148.pdf $18 / 02 / 2019$

Fuente M.E. y Barkin D. (2013). La minería como factor de desarrollo en la Sierra Juárez de Oaxaca: Una valoración ética. Probl. Desarrollo 44 (172), 123-144. DOI: $10.1016 / \mathrm{S} 0301-7036(13) 71865-0$

Genchi G., Sinicropi M.S., Carocci A., Lauria G. y Catalano A. (2017). Mercury exposure and heart diseases. Int. J. Environ. Res. Public Health 14 (1), 74.

DOI: 10.3390/ijerph14010074

Graeme K.A. y Pollack C.V. (1998). Heavy metal toxicity, part I: Arsenic and mercury. J. Emerg. Med. 16 (1), 45-56. DOI: $10.1016 / \mathrm{S} 0736-4679(97) 00241-2$

Grandjean P., Weihe P., White R.F., Debes F., Araki S., Yokoyama K., Murata K., Sørensen N., Dahl R. y Jørgensen P.J. (1997). Cognitive deficit in 7-year-old children with prenatal exposure to methylmercury. Neurotoxicol. Teratol. 19 (6), 417-428.

DOI: $10.1016 / \mathrm{S} 0892-0362(97) 00097-4$

Guevara B.X. (2016). La inversión extranjera directa en la minería en México: el caso del oro. Análisis Económico 31 (77), 85-113.

Ibrahim D., Froberg B., Wolf A. y Rusyniak D.E. (2006). Heavy metal poisoning: Clinical presentations and pathophysiology. Clin. Lab. Med. 26 (1), 67-97. DOI: $10.1016 /$ j.cll.2006.02.003

INECC (2016). Metodología de valoración económica del daño del medio ambiente por contingencias del sector minero. Instituto Nacional de Ecología y Cambio Climático, Ciudad de México, México, 136 pp.

INEGI (2015). Banco de indicadores. Instituto Nacional de Estadística y Geografía [en línea]. https://www.inegi. org.mx/app/indicadores/ 18/02/2019

Lindsay W.L. y Norvell W.A. (1978). Development of a DTPA soil zinc, iron, manganese and copper. Soil Sci. Soc. Am. J. 42 (3), 421-428.

DOI: $10.2136 /$ sssaj1978.03615995004200030009x

Mancilla-Villa O., Ortega-Escobar H., Ramírez-Ayala C., Uscanga-Mortera E., Ramos-Bello R. y ReyesOrtigoza A. (2012). Metales pesados totales y arsénico en el agua para riego de Puebla y Veracruz, México. Rev. Int. Contam. Ambie. 28 (1), 39-48. 
Martín F. y Gutiérrez M. (2010). Estudio comparativo de la peligrosidad de jales en dos zonas mineras localizadas en el sur y centro de México. B. Soc. Geol. Mex. 62 (1), 43-53.

DOI: 10.18268/BSGM2010v62n1a3

Martínez C., Prieto A., García L., Quero A., González S. y Casan P. (2010). Silicosis, una enfermedad con presente activo. Arch. Bronconeumol. 46 (2), 97-100. DOI: 10.1016/j.arbres.2009.07.008

Moreno E. (2010). Recuperación de suelos mineros contaminados con arsénico mediante fitotecnologías. Tesis de Doctorado. Departamento de Química Agrícola, Universidad Autónoma de Madrid. Madrid, España, $207 \mathrm{pp}$.

Moreno R., Téllez J. y Monroy M.G. (2012). Influencia de los minerales de los jales en la bioaccesibilidad de arsénico, plomo, zinc y cadmio en el distrito minero Zimapán, México. Rev. Int. Contam. Ambie. 28 (3), 203-218.

Needleman H. (2004). Lead poisoning. Annu. Rev. Med. 55, 209-222.

DOI: 10.1146/annurev.med.55.091902.103653

Ordóñez B.R., Ruiz L. y Mora R. (2003). Investigación epidemiológica sobre niveles de plomo en la población infantil y en el medio ambiente domiciliario de Ciudad Juárez, Chihuahua, en relación con una fundición de El Paso, Texas. Rev. Salud Pública Méx., 45 (supl. 2), 281-295.

Ortiz-Ortiz E., García-Nieto E., Juárez-Santacruz L., Gómez-Camarillo M.A., García-Gallegos E. y LimónHuitrón P. (2017). Lead exposure: Pottery impact in Tlaxcala, México. Rev. Int. Contam. Ambie. 33 (1), 57-64. DOI: 10.20937/RICA.2017.33.01.05

Parga J.R. y Carrillo F.R. (1996). Avances en los métodos de recuperación de oro y plata de minerales refractarios. Rev. Metal. Madrid 32 (4), 254-261.

DOI: 10.3989/revmetalm.1996.v32.i4.907

Peters B.A., Hall M.N., Liu X., Neugut Y.D., Pilsner J.R., Levy D., Ilievski V., Slavkovich V., Islam T., Factor-Litvak P., Graziano J.H. y Gamble M.V. (2014). Creatinine, arsenic metabolism, and renal function in an arsenic-exposed population in Bangladesh. Plos One 9 (12), 1-22. DOI: 10.1371/journal.pone. 0113760

Poma P.A. (2008). Intoxicación por plomo en humanos. An. Fac. Med. 69 (2), 120-126.

DOI: 10.15381/anales.v69i2.1155

Puga S., Sosa M., Lebgue T., Quintana C. y Campos A. (2006). Contaminación por metales pesados en suelo provocada por la industria minera. Ecol. Apl. 5 (1-2), 149-155. DOI: 10.21704/rea.v5i1-2.329

Ramírez A.V. (2005). El cuadro clínico de la intoxicación ocupacional por plomo. An. Fac. Med. 66 (1), 57-70. DOI: 10.15381/anales.v66i1.1352
Ramos-Gómez M., Avelar J., Medel-Reyes A., Yamamoto L., Godínez L., Ramírez M., Guerra R. y Rodríguez F. (2012). Movilidad de metales en jales procedentes del distrito minero de Guanajuato, México. Rev. Int. Contam. Ambie. 28 (1), 49-59.

Rees D. y Murray J. (2007). Silica, silicosis and tuberculosis. Int. J. Tuberc. Lung. Dis. 11 (5), 474-484.

Romero F.M., Armienta M.A., Gutiérrez M.E. y Villaseñor G. (2008). Factores geológicos y climáticos que determinan la peligrosidad y el impacto ambiental de jales mineros. Rev. Int. Contam. Ambie. 24 (2), 43-54.

Salinas E., Rivera I., Carrillo F.R., Patiño F., Hernández J. y Hernández L.E. (2004). Mejora del proceso de cianuración de oro y plata, mediante la preoxidación de minerales sulfurosos con ozono. Rev. Soc. Quím. Méx. 48 (4), 315-320.

Sánchez A. (1993). Territorio y minería en Oaxaca: la exploración de minerales metálicos al inicio de los noventa. Investigaciones Geográficas 1 (26), 65-89. DOI: $10.14350 /$ rig. 59018

SEMARNAT (1996). Norma Oficial Mexicana NOM-001SEMARNAT-1996. Establece los límites máximos permisibles de contaminantes en las descargas de aguas residuales en aguas y bienes nacionales. Secretaría de Medio Ambiente, Recursos Naturales y Pesca, Diario Oficial de la Federación, 23 de abril.

SEMARNAT (2003). Norma Oficial Mexicana NOM141- SEMARNAT-2003. Requisitos para la caracterización del sitio, proyecto, construcción, operación y postoperación de presas de jales. Secretaría de Medio Ambiente, Recursos Naturales y Pesca, Diario Oficial de la Federación, 14 de enero.

SEMARNAT (2004). Norma Oficial Mexicana NOM147- SEMARNAT-2004. Criterios para determinar las concentraciones de remediación de suelos contaminados por arsénico, bario, berilio, cadmio, cromo hexavalente, mercurio, níquel, plata plomo, selenio, talio y/o vanadio. Secretaría de Medio Ambiente, Recursos Naturales y Pesca, Diario Oficial de la Federación, 2 de marzo.

SIAM (2015). Portafolio de proyectos. Sistema de Administración Minera [en línea]. http://www.siam. economia.gob.mx/es/siam/p_Portafolio_de_Proyectos $18 / 02 / 2019$

Sigüenza S. (1996). Minería y comunidad indígena: el mineral de Natividad, Ixtlán, Oaxaca (1900-1940). Centro de Investigaciones y Estudios Superiores en Antropología Social, Ciudad de México, México, 157 pp.

Simón M., Ortiz I., García I., Fernández E., Fernández J., Dorronsoro C. y Aguilar J. (1999). Pollution of soil by the toxic spill of a pyrite mine (Aznalcóllar, Spain). Sci. Total Environ. 242 (1-3), 105-115. DOI: $10.1016 / \mathrm{S} 0048-9697(99) 00378-2$ 
Teodoro M. (2014). Estudio de la comunidad vegetal presente en sitios con residuos de minas de plata y su capacidad fitorremediadora. Tesis de Maestría. Centro Interdisciplinario de Investigación para el Desarrollo Integral Regional Unidad Oaxaca, Instituto Politécnico Nacional, Santa Cruz Xoxocotlán, Oaxaca, México, $53 \mathrm{pp}$.

Warren L.A. y Haack E.A. (2001). Biogeochemical controls on metal behavior in freshwater environments. Earth-Sci. Rev. 54 (4), 261-320.

DOI: $10.1016 / \mathrm{S} 0012-8252(01) 00032-0$
Xu J., Lian L.J., Wu C., Wang X.F., Fu W.Y. y Xu L.H. (2008). Lead induces oxidative stress, DNA damage and alteration of p53: Bax and Bcl-2 expressions in mice. Food Chem. Toxicol. 46 (5), 1488-1494. DOI: $10.1016 /$ j.fct.2007.12.016

Zhao L.Y.L., Schulin R., Wenig L.P. y Nowack B. (2007). Coupled mobilization of dissolved organicbmatter and metals $(\mathrm{Cu}$ and $\mathrm{Zn})$ in soil columns. Geochim. Comsochim. Ac. 71 (14), 3407-3418.

DOI: $10.1016 /$ j.gca.2007.04.020 